\title{
An evaluation of the Lyapunov characteristic exponent of chaotic continuous systems
}

\author{
Sandra Rugonyi and Klaus-Jürgen Bathe ${ }^{* \dagger}$ \\ Mechanical Engineering Department, Massachusetts Institute of Technology, Cambridge, MA 02139, U.S.A.
}

\begin{abstract}
SUMMARY
A procedure to calculate the Lyapunov characteristic exponent of the response of structural continuous systems, discretized using finite element methods, is proposed. The Lyapunov characteristic exponent can be used to characterize the asymptotic stability of the system dynamic response, and it is frequently employed to identify a chaotic behaviour. The proposed procedure can also be used in the stability characterization of fluid-structure interaction systems in which the focus of the analysis is on the behaviour of the structural part. Copyright (c) 2002 John Wiley \& Sons, Ltd.
\end{abstract}

KEY WORDS: stability analysis; Lyapunov characteristic exponent; chaotic behaviour; non-linear dynamics; dynamic stability

\section{INTRODUCTION}

The study of the non-linear behaviour of systems is nowadays essential in almost every field of engineering and science. The large variety of behaviours that non-linear systems can exhibit makes the response solution difficult to find in many situations, even when numerical methods are available.

In this paper we are interested in assessing the stability of motions or dynamic stability of systems. To this end, the concept of Lyapunov stability is used. Consider an initial perturbation, $\mathbf{y}_{0}$, to the system response at time $t_{0}^{*}$. The motion is said to be stable if for two real, reasonably small, numbers $\varepsilon, \delta$, for each $\varepsilon>0$ there exists a $\delta>0$ such that if $\left\|\mathbf{y}_{0}\right\|<\delta$ then $\|\mathbf{y}(t)\|<\varepsilon$ for all $t>t_{0}^{*}$. In addition, the motion is quasi-asymptotically stable if $\left\|\mathbf{y}_{0}\right\|<\delta$ and $\lim _{t \rightarrow \infty}\|\mathbf{y}(t)\|=0$. The motion is said to be unstable if it is not stable. A chaotic motion is an asymptotic dynamically unstable response, since, once chaotic motion occurs, perturbations to the system response grow exponentially fast.

In engineering practice, an unstable response is usually undesirable, and therefore it becomes important to characterize the dynamic stability of the response of systems. Such characterization can be performed by calculating the response Lyapunov characteristic exponent, which

*Correspondence to: Klaus-Jürgen Bathe, Mechanical Engineering Department, Massachusetts Institute of Technology, 77 Massachusetts Avenue, Cambridge, MA 02139, U.S.A.

†E-mail: kjb@mit.edu

Received 8 August 2001

Copyright (c) 2002 John Wiley \& Sons, Ltd.

Revised 23 January 2002

Accepted 8 March 2002 
measures the divergence of nearby trajectories in the system phase space (it is associated with the growth of infinitesimally small perturbations to the response). The Lyapunov characteristic exponent is not only helpful in identifying the stability behaviour of the system in consideration, once transient effects have decayed, but it also provides a measure of the stochastic characteristics of a chaotic behaviour. The calculation of the Lyapunov characteristic exponent is a valuable tool in the study of dynamical systems, since the stability of a system response is not always obvious by 'simply only looking' at the system behaviour.

Numerous works have been published on the theory of non-linear dynamical systems and chaotic behaviours, and on the computation of the Lyapunov characteristic exponent of discrete and relatively small order systems of equations, see for example References [1-6]. However, the chaotic behaviour of continuous systems has only been studied using experimental investigations and/or modal reduction techniques, see for example References [7-11]; and using finite element methods but treating the numerically calculated response in the same way as the output of an experimental study $[12,13]$. There seems to be no previous work in which the system Lyapunov characteristic exponent is calculated by discretizing the continuous equations of motion and applying a perturbation to all resulting discrete degrees of freedom. The reason is that the equations of motion resulting from the discretization of a continuous system, employing for example finite element methods, present significant differences compared to the discrete systems of equations studied in the literature. The most important differences are: (i) the discretization of a continuous system results in a large number of degrees of freedom (variables) to solve for; and (ii) completely different time scales are present in the discretized equations of motion of a continuous system. Consequently, the procedures proposed to compute the Lyapunov characteristic exponent of discrete non-dimensional systems, see for instance References [4-6], cannot be directly employed when discretized structural continuous problems are considered.

In this paper, a procedure to calculate the Lyapunov characteristic exponent of continuous structural systems and fluid-structure interaction problems, in which the structural behaviour is the focus of the analysis, is presented. The procedure is based on the perturbation of all the degrees of freedom of continuous systems discretized using finite element methods. In addition, the use of a local (temporal) stability indicator is also suggested. The indicator can be used to capture dynamic instabilities in the system shortly after they occur.

\section{LYAPUNOV CHARACTERISTIC EXPONENTS}

In this section, the definition and properties of Lyapunov characteristic exponents are briefly reviewed. Also, the numerical calculation of the maximum Lyapunov characteristic exponent (of order one) is briefly discussed for the case of non-dimensional discrete systems of firstorder ordinary differential equations in time.

For simplicity of exposition, it will be assumed throughout the paper that Cartesian coordinates are employed.

\subsection{Definition and properties of Lyapunov characteristic exponents}

Consider the following non-dimensional system of equations:

$$
\dot{\mathbf{x}}=\mathbf{f}(\mathbf{x})
$$


with initial conditions

$$
\mathbf{x}\left(t_{0}\right)=\mathbf{x}_{0}
$$

where $\mathbf{x} \in \Re^{n}$ is the vector of state variables for the system, $\mathbf{f}$ is a non-linear vector valued function, and $t$ represents time.

The system of equations (1) is called autonomous since the function $\mathbf{f}$ does not depend on time. In case $\mathbf{f}$ depends on time, i.e. $\mathbf{f}(\mathbf{x}, t)$, the equations of motion can be converted into an autonomous system of equations by performing a change of variables, $x_{n+1}=t$ and therefore $\dot{x}_{n+1}=1$, where $n$ is the number of state variables for the system in consideration. In this way, time is included as a state variable, and the techniques developed for autonomous systems can be employed in the analysis of non-autonomous systems.

Assume that $\mathbf{f}$ is a continuously differentiable $\left(C^{1}\right)$ function that generates a flow $\phi_{t}$ defined on a differentiable manifold, $M$. Then Equation (1) has unique solutions for all values of $t$, see for example Reference [1]. We are interested in the asymptotic behaviour (as $t \rightarrow \infty$ ) of the differential flow $\mathrm{d} \phi_{t}$.

This problem is related to the asymptotic behaviour of infinitesimal perturbations $\mathbf{y}(t)$ to the reference trajectory $\mathbf{x}_{r}(t)$, the solution of Equations (1) and (2).

Given a perturbation $\mathbf{y}$ to the reference trajectory $\mathbf{x}_{r}$, the perturbed trajectory is $\mathbf{x}=\mathbf{x}_{r}+\mathbf{y}$, where it is assumed that $\|\mathbf{y}\| \ll\left\|\mathbf{x}_{r}\right\|$. The equation that describes the evolution of infinitesimal perturbations to the reference trajectory is

$$
\dot{\mathbf{y}}=\left(\frac{\partial \mathbf{f}}{\partial \mathbf{x}}\right)_{\mathbf{x}=\mathbf{x}_{r}} \mathbf{y}
$$

where $\partial \mathbf{f} / \partial \mathbf{x}$ is the Jacobian matrix of the vector function $\mathbf{f}(\mathbf{x})$.

A solution of Equation (3) exists and is unique for every initial condition $\mathbf{y}\left(t_{0}^{*}\right)=\mathbf{y}_{0}$, and the perturbation can be expressed as ${ }^{\ddagger}$

$$
\mathbf{y}(t)=\mathbf{\Phi}\left(t, t_{0}^{*}\right) \mathbf{y}_{0}
$$

where the matrix $\boldsymbol{\Phi}\left(t, t_{0}^{*}\right)$ is the linearized flow matrix, a linear map of the tangent space of $\mathbf{f}\left(\mathbf{x}_{r}\right)$ at time $t_{0}^{*}, E_{0}$, onto the tangent space of $\mathbf{f}\left(\mathbf{x}_{r}\right)$ at time $t, E_{t}$. The following relations, for the mapping $\boldsymbol{\Phi}$, are satisfied,

$$
\begin{aligned}
& \boldsymbol{\Phi}\left(t_{2}, t_{0}^{*}\right)=\mathbf{\Phi}\left(t_{2}, t_{1}\right) \boldsymbol{\Phi}\left(t_{1}, t_{0}^{*}\right) \\
& \boldsymbol{\Phi}\left(t_{n}, t_{n}\right)=\mathbf{I}
\end{aligned}
$$

where $t_{2}>t_{1}>t_{0}^{*}$, and $\mathbf{I}$ is the identity matrix. Note that, since the matrix $\boldsymbol{\Phi}$ is obtained from Equation (3) and the Jacobian matrix changes as a function of time, $\boldsymbol{\Phi}$ depends on the specific times considered and on the reference trajectory.

Assume that

$$
\lim _{t \rightarrow \infty} \frac{1}{t} \ln \left\|\mathbf{\Phi}\left(t, t_{0}^{*}\right)\right\|<\infty
$$

\footnotetext{
¥Note that the initial time for the equations of motion, $t_{0}$, is not necessarily equal to the initial time for the perturbation equations, $t_{0}^{*}$, which is usually selected such that transient effects have decayed. 


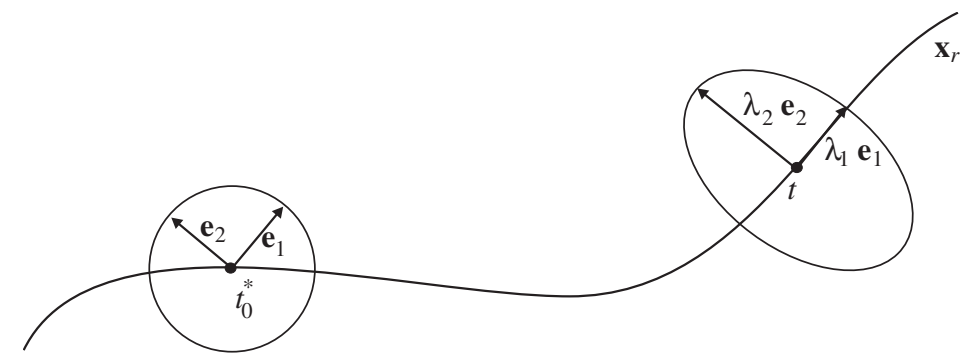

Figure 1. Graphical representation of the evolution of perturbations in a twodimensional phase space. The vectors $\mathbf{e}_{1}$ and $\mathbf{e}_{2}$ change directions as the value of $t$ increases, and it is assumed that $\left\|\mathbf{e}_{1}\right\|=\left\|\mathbf{e}_{2}\right\|=1$.

where the norm of the matrix $\boldsymbol{\Phi}$ is usually defined as $\|\boldsymbol{\Phi}\|=\max \left[\lambda\left(\sqrt{\boldsymbol{\Phi}^{\mathrm{T}} \boldsymbol{\Phi}}\right)\right]$ and $\lambda(\mathbf{A})$ represents the eigenvalues of the matrix A. Then, a one-dimensional Lyapunov characteristic exponent of the response of the non-linear system (1), with initial conditions (2), is defined by $[5,14]$ :

$$
\chi\left(\mathbf{y}_{0}, \mathbf{x}_{r}\right)=\limsup _{t \rightarrow \infty} \frac{1}{t} \ln \left(\frac{\|\mathbf{y}(t)\|}{\left\|\mathbf{y}_{0}\right\|}\right)
$$

where the supremum is taken since otherwise the function can be oscillatory and the limit not be defined.

Oseledec considered the conditions for which the limit exists [14]. For the system of Equations (1), if $\|\partial \mathbf{f} / \partial \mathbf{x}\|$ is Lebesgue integrable, the limit exists and then

$$
\chi\left(\mathbf{y}_{0}, \mathbf{x}_{r}\right)=\lim _{t \rightarrow \infty} \frac{1}{t} \ln \left(\frac{\|\mathbf{y}(t)\|}{\left\|\mathbf{y}_{0}\right\|}\right)
$$

A geometrical interpretation of $\chi\left(\mathbf{y}_{0}, \mathbf{x}_{r}\right)$ can be established. Imagine an $n$-dimensional unit sphere $D$ in the tangent space of $\mathbf{f}(\mathbf{x})$ at $\mathbf{x}_{r}\left(t_{0}^{*}\right), E_{0}$ ( $D$ can be thought of as an $n$-dimensional unit sphere of perturbed initial conditions). The image of $D$ in the tangent space $E_{t}$ at $\mathbf{x}_{r}(t)$ is given by the mapping $\boldsymbol{\Phi}\left(t, t_{0}^{*}\right)$ and is in general an ellipsoid, see Figure 1. It is possible to associate each ellipsoid semiaxis with a coefficient of expansion (also called a Lyapunov characteristic number), $\lambda_{i}$. Furthermore, we can associate each coefficient of expansion with a Lyapunov characteristic exponent, $\chi_{i}$, and therefore $n$ Lyapunov characteristic exponents are obtained, where $n$ is the dimension of the tangent space,

$$
\chi_{i}=\lim _{t \rightarrow \infty} \frac{1}{t} \ln \lambda_{i} \quad \text { with } i=1,2, \ldots, n
$$

We arrange these in descending order,

$$
\chi_{1} \geqslant \chi_{2} \geqslant \cdots \geqslant \chi_{n}
$$

It can be shown that except in the case of steady-state solutions, at least one of the Lyapunov characteristic exponents is zero $[5,15]$.

In addition, it can be shown [2,5] that the value in Equation (8) does not depend on the initial perturbation vector selected, and in a numerical solution the maximum Lyapunov 
characteristic exponent is obtained. This is because at each time step $\mathbf{y}$ tends to grow more in the direction of the eigenvector of $\boldsymbol{\Phi}$ that corresponds to the eigenvalue with largest real part. As a consequence, after a sufficient number of time steps, the vector $\mathbf{y}$ is 'aligned' with the direction associated with $\chi_{1}$, so that $\chi_{1}$ is obtained from the calculation.

Furthermore, all trajectories, $\mathbf{x}_{r}$, that start in the basin of attraction of an attracting set will give the result

$$
\chi\left(\mathbf{y}_{0}, \mathbf{x}_{r}\right)=\chi_{1}
$$

and we call $\chi$ the Lyapunov characteristic exponent (LCE) of the response in consideration.

\subsection{Numerical calculation of the LCE of discrete systems}

The following procedure has been employed to calculate the LCE of discrete dynamical systems [5]. The procedure has been used with non-dimensional first-order ordinary differential equations in time (and maps) of only a few degrees of freedom.

Since in Equation (3) the Jacobian matrix of $\mathbf{f}(\mathbf{x})$ has to be evaluated at $\mathbf{x}_{r}(t)$, both Equations (1) and (3) have to be solved to calculate the system LCE.

Without loss of generality, a random initial perturbation $\mathbf{y}_{0}$ is chosen such that $\left\|\mathbf{y}_{0}\right\|=1$; and a fixed time step $\Delta t$ is employed, such that $t_{n}=t_{0}^{*}+n \Delta t$.

The evolution of the perturbation vector at each time step, $t_{n}$, can be calculated by discretizing Equation (3) in time and solving for $\mathbf{y}\left(t_{n}\right)=\mathbf{y}_{n}$.

The perturbation vector $\mathbf{y}_{n}$ may grow (exponentially fast) and hence computer overflow can occur in the numerical calculation. To avoid the overflow, the vector is normalized at every time step (or after a certain number of time steps) and the calculation proceeds with the normalized vector. Denoting by $d_{n}$ the norm of the obtained perturbation vector at each time step, it is evident from Equations (4) and (5) that after $n$ time steps

$$
\left\|\mathbf{y}_{n}\right\|=d_{n} d_{n-1} \cdots d_{1}
$$

An approximation to the LCE can be calculated, at each time step, as follows:

$$
k_{n}=\frac{1}{t_{n}} \ln \left\|\mathbf{y}_{n}\right\|=\frac{1}{t_{n}} \sum_{i=1}^{n} \ln \left(d_{i}\right)
$$

The LCE is then calculated as the limit

$$
\chi=\lim _{n \rightarrow \infty, \Delta t \rightarrow 0} k_{n}
$$

In practice, a good approximation to the value of the LCE is obtained when $k_{n}$ reaches a constant value as $n$ increases.

\section{NUMERICAL EVALUATION OF THE LCE OF CONTINUOUS SYSTEMS}

In this section the calculation of the response LCE of continuous systems is presented. The systems considered are structural problems and fluid-structure interaction problems in which the focus of the analysis is on the behaviour of the structural part of the system. A local temporal stability indicator, which can be used with both types of problems, is also presented. 
It is assumed that the numerical scheme employed to discretize the continuous equations of motion is 'stable' (i.e. no numerical instabilities are present in the calculated system response) and that the computed response accurately captures the nature of the system behaviour. Thus, we will concentrate throughout this work on the system's physical dynamic instabilities as represented by the system mathematical model employed.

\subsection{Structural problems}

The differential equations that govern the dynamic behaviour of a solid medium are

$$
\rho \ddot{\mathbf{u}}=\nabla \cdot \tau+\mathbf{f}^{B}
$$

with boundary conditions

$$
\begin{array}{rr}
\mathbf{u}=\mathbf{u}^{S} & \text { on } S_{u}^{S} \\
\tau \mathbf{n}^{S}=\mathbf{t}^{S} & \text { on } S_{f}^{S}
\end{array}
$$

and initial conditions

$$
\mathbf{u}\left(t_{0}\right)=\mathbf{u}_{0}
$$

In the above equations, $\rho$ is the structural mass density, $\mathbf{u}$ is the vector field of structural displacements, $\tau$ is the Cauchy stress tensor of the structure, $\mathbf{f}^{B}$ is the vector of applied body forces, $S_{u}^{S}$ is the part of the structural boundary with imposed displacements $\mathbf{u}^{s}, S_{f}^{S}$ is the part of the structural boundary with imposed tractions $\mathbf{t}^{s}$, and $\mathbf{n}^{s}$ is the outward unit normal vector to the solid surface.

Equation (15) is non-linear if geometric non-linearities are present (when the deformations of the solid are significant) or if the material is non-linear (that is, when $\tau$ is a non-linear function of $\mathbf{u}$ and/or $\dot{\mathbf{u}}$ ).

Discretizing these equations using finite element methods, the following discrete equations are obtained for time $t$ :

$$
{ }^{t} \mathbf{F}={ }^{t} \mathbf{R}_{S}
$$

where ${ }^{t} \mathbf{F}$ is the vector of nodal point forces corresponding to the internal element stresses and inertial forces and ${ }^{t} \mathbf{R}_{s}$ is the vector of discretized loads applied to the body (see Reference [16] for a detailed description).

The displacements and velocities of the structural nodal degrees of freedom (and possibly the time) form the finite element discretized phase space of the problem in consideration.

If $\tilde{\mathbf{u}}$ corresponds to perturbations in the displacements, the linearized equations that describe the evolution of perturbations, neglecting dissipative terms, can be written as

$$
\mathbf{M} \ddot{\tilde{u}}+\mathbf{K u}=\mathbf{0}
$$

with initial conditions,

$$
\begin{aligned}
\tilde{\mathbf{u}}\left(t_{0}^{*}\right) & =\tilde{\mathbf{u}}_{0} \\
\dot{\tilde{\mathbf{u}}}\left(t_{0}^{*}\right) & =\dot{\tilde{\mathbf{u}}}_{0}
\end{aligned}
$$

where $\mathbf{M}$ and $\mathbf{K}$ are the mass and tangent stiffness matrix of the problem, respectively. 
Note that for a continuum model the perturbations must be compatible with the boundary conditions at all times. Thus, the boundary conditions for the perturbations are specified to be

$$
\begin{array}{rr}
\tilde{\mathbf{u}}=\mathbf{0} & \text { on } S_{u}^{S} \\
\tilde{\tau} \mathbf{n}^{S}=\mathbf{0} & \text { on } S_{f}^{S}
\end{array}
$$

where $\tilde{\tau}=\tau(\mathbf{u}+\tilde{\mathbf{u}})-\tau(\mathbf{u})$ is equal to the difference between the stress tensor of the perturbed problem, $\tau(\mathbf{u}+\tilde{\mathbf{u}})$, and the stress tensor of the reference problem, $\tau(\mathbf{u})$.

Equation (20) could be easily re-written in the form of Equation (1) with $\mathbf{y}^{\mathrm{T}}=(\tilde{\mathbf{u}} \dot{\tilde{\mathbf{u}}})$. However, it is more effective to solve directly the second-order differential equations in time using, for instance, the Newmark method of time integration, see Reference [16]. Otherwise, the number of equations to solve increases from $N$ to $2 N$, where $N$ is the number of degrees of freedom for the problem considered.

In order to calculate the LCE of the discretized continuous system, the following procedure is proposed (and discussed in Section 4):

1. Choose an initial perturbation vector such that $d_{0}=\left\|\tilde{\mathbf{u}}_{0}\right\| / L=1$; $\tilde{\mathbf{u}}_{0}$ satisfies the perturbation problem boundary conditions but is otherwise arbitrary. Set $\hat{\mathbf{u}}_{0}$ equal to $\tilde{\mathbf{u}}_{0} / L$ and set $\dot{\hat{\mathbf{u}}}_{0}$ equal to zero or equal to an arbitrary vector. Here $L$ is a characteristic length of the problem in consideration.

2. Given the (normalized) 'initial' conditions $\hat{\mathbf{u}}_{n}$ and $\dot{\hat{\mathbf{u}}}_{n}$ at each time step, calculate the accelerations that satisfy Equation (20), at time $t_{n}$.

3. Discretize Equation (20) in time, and solve for $\tilde{\mathbf{u}}_{n+1}^{*}$ and $\dot{\tilde{\mathbf{u}}}_{n+1}^{*}$, which are the nodal point displacements and velocities, for time $t_{n+1}$, obtained from Equation (20), using $\hat{\mathbf{u}}_{n}$ and $\dot{\hat{\mathbf{u}}}_{n}$ as 'initial' conditions in the time step calculation. ${ }^{\S}$

4. Obtain the norm of the perturbation as

$$
d_{n+1}=\left\|\tilde{\mathbf{u}}_{n+1}^{*}\right\|
$$

5. Normalize the perturbation,

$$
\hat{\mathbf{u}}_{n+1}=\frac{\tilde{\mathbf{u}}_{n+1}^{*}}{d_{n+1}} ; \quad \dot{\hat{\mathbf{u}}}_{n+1}=\frac{\dot{\tilde{\mathbf{u}}}_{n+1}^{*}}{d_{n+1}}
$$

6. Calculate $k_{n+1}$ as,

$$
k_{n+1}=\frac{1}{t_{n+1}} \sum_{i=1}^{n+1} \ln d_{i}
$$

and advance to the next time step, by repeating steps $2-6$ with $\hat{\mathbf{u}}_{n+1}$ and $\dot{\hat{\mathbf{u}}}_{n+1}$ as the normalized 'initial' conditions.

The system response LCE is obtained from

$$
\chi=\limsup _{n \rightarrow \infty} k_{n}
$$

$\overline{\S \text { Note that here }} \tilde{\mathbf{u}}_{n+1}^{*}=\boldsymbol{\Phi}\left(t_{n+1}, t_{n}\right) \hat{\mathbf{u}}_{n}=d_{n+1} \hat{\mathbf{u}}_{n+1}$. 
The same time discretization (and time step) should be used to calculate the evolution of perturbations and to solve the discrete equations of motion of the system (19). The advantage is that exactly the same (left-hand side) matrices are used in the calculation of the first iteration of the non-linear problem (when using for instance the Newton-Raphson scheme) and in the calculation of the perturbation evolution at each time step. As a consequence, the coefficient matrix obtained has to be factorized only once, and can then be used for both the system response and perturbation calculation.

\subsection{Fluid-structure interaction problems}

Different types of fluid-structure interaction (FSI) analyses can be performed depending on the model chosen to describe the fluid behaviour $[17,18]$. The procedure described below can be used with any model. However, the specific example solved in Section 5 corresponds to the case in which the fluid is modelled using the (almost) incompressible Navier-Stokes equations of motion, and the solution is obtained using the coupling and condensation procedure described in Reference [17].

When analysing FSI problems, it may not be important to characterize the fluid flow stability, since turbulence, which is an unstable behaviour of the fluid flow, is in many situations a desired response for the flow. However, instabilities of the structure, resulting from the interaction with the fluid flow, are undesirable, and therefore a dynamic stability analysis of the structure is essential.

For the structure, the fluid flow interaction is equivalent to a forcing term at the fluidstructure interface. This forcing term depends on the fluid pressure and velocity, $\mathbf{p}$ and $\mathbf{v}$, and on the displacement of the fluid-structure interface, $\mathbf{u}^{I}$,

$$
{ }^{t} \mathbf{F}={ }^{t} \mathbf{R}_{s}^{S}+{ }^{t} \mathbf{R}_{s}^{I}\left(\mathbf{u}^{I}, \mathbf{v}, \mathbf{p}\right)
$$

where ${ }^{t} \mathbf{R}_{s}^{I}$ represents the forces applied to the structural boundary exerted by the fluid and ${ }^{t} \mathbf{R}_{s}^{S}$ represents all other applied forces.

From Equation (27), the equation for the evolution of structural perturbations in the system can be obtained as follows:

$$
\mathbf{M} \ddot{\tilde{\mathbf{u}}}+\mathbf{K} \tilde{\mathbf{u}}-\left(\frac{\partial \mathbf{R}_{s}^{I}}{\partial \mathbf{u}^{I}}\right)_{t} \tilde{\mathbf{u}}-\left(\frac{\partial \mathbf{R}_{s}^{I}}{\partial \mathbf{v}}\right)_{t} \tilde{\mathbf{v}}-\left(\frac{\partial \mathbf{R}_{s}^{I}}{\partial \mathbf{p}}\right)_{t} \tilde{\mathbf{p}}=\mathbf{0}
$$

Thus, it is important to identify and consider, at each time step, the forces exerted from the fluid onto the structure in the calculation of the evolution of the perturbations.

Alternatively, the complete linearized equations for the fluid flow coupled with the structure can be considered to compute the evolution of perturbations. However, since the main interest lies in the analysis of the behaviour of the structural/solid part of the problem, only the displacements of the structural degrees of freedom are used in the calculation of the norm of the perturbation vector, $d_{n}$, as described in Section 3.1. In this way, while only the growth/decay of structural perturbations are considered and specifically followed, the effects of these perturbations in both the structural and fluid domains are considered. This approach was used in the computation of the LCE of the fluid-structure interaction system presented in Section 5. 


\subsection{Local stability indicator}

It is frequently important to know the local dynamic stability of the system, and the specific instant at which the system dynamic behaviour changes from stable to unstable. This information is not contained in the response LCE, which is a measure of the asymptotic divergence of nearby trajectories in phase space. However, it is possible to use the value of the accumulated perturbation norm, $k_{n} t_{n}$, at each time step, to assess the local stability of the system response. Defining $\tilde{d}\left(t_{n}\right)=d_{n} d_{n-1} \cdots d_{1}$, which measures the divergence or convergence of trajectories in the phase space (i.e. the growth/decay of infinitesimal perturbations), an instability can be captured by the value of $\ln \tilde{d}\left(t_{n}\right)=k_{n} t_{n}$. Note however that the current values of $d_{n}$ or $\ln \tilde{d}\left(t_{n}\right)$ alone do not provide information about the response local stability since the values of $d_{n}$ oscillate. The overall system stability behaviour is however identified by studying the growth/decay of the maximum values of $\ln \tilde{d}\left(t_{n}\right)$. The average slope of the curve of $\ln \tilde{d}\left(t_{n}\right)$ as a function of time indicates the local stability of the response.

It is important to mention that the behaviour of $\ln \tilde{d}\left(t_{n}\right)$ is representative of the system response only after transient effects in the calculation of the perturbation vector have decayed.

The local stability indicator is then equivalent to performing a traditional linearized stability analysis, except that it is not necessary to calculate the eigenvalues associated with the behaviour of small perturbations to the system response.

\section{THE EFFECT OF VELOCITIES IN THE LCE CALCULATION}

For a structural discretized problem, the relevant perturbed variables in phase space are the system nodal point displacements and velocities, and therefore the discretized phase space is in $\Re^{2 N}$. However, displacements and velocities have different units, and a non-dimensionalization must be performed to calculate the norm of the perturbation vector. We use a characteristic length $L$, and a characteristic velocity $V$ to non-dimensionalize the perturbations,

$$
\tilde{d}(t)=\sqrt{\frac{\|\tilde{\mathbf{u}}\|^{2}}{L^{2}}+\frac{\|\dot{\tilde{\mathbf{u}}}\|^{2}}{V^{2}}}
$$

Note that since $\tilde{\mathbf{u}}$ and $\dot{\tilde{\mathbf{u}}}$ are not normalized, $\tilde{d}\left(t_{n}\right)=d_{n} d_{n-1} \cdots d_{1}$, as defined before (although here velocity perturbations are included as well).

When using Equation (29) to calculate approximations to the LCE,

$$
k_{n}=\frac{1}{t_{n}} \ln \tilde{d}\left(t_{n}\right)
$$

it is observed that the values of $k_{n}$ can grow as a function of time, $t_{n}$, for very large periods of time even when a dynamically stable problem is considered. The use of proper values of the problem characteristic length and velocity, $L$ and $V$, in Equation (29) is essential to the procedure. It is natural to use $V=\hat{\omega} L$, with $\hat{\omega}$ to be selected, in which case $L$ has the role of a scaling constant.

To elucidate how to choose an appropriate value of $\hat{\omega}$ let us first consider a very simple problem: a single degree of freedom harmonic oscillator (which cannot become chaotic or 
unstable),

$$
\ddot{x}+\omega^{2} x=0
$$

with $x$ the displacement and $\omega$ the frequency of oscillation.

The equation for the evolution of the perturbation, $\tilde{x}$, is

$$
\ddot{\tilde{x}}+\omega^{2} \tilde{x}=0
$$

Using $V=\omega L$ in Equation (29) for the harmonic oscillator

$$
\tilde{d}(t)=\sqrt{\frac{\tilde{x}^{2}}{L^{2}}+\frac{\dot{\tilde{x}}^{2}}{\omega^{2} L^{2}}}=1
$$

assuming that $d_{0}=1$. The response LCE is calculated as

$$
\chi=\lim _{t \rightarrow \infty} \frac{1}{t} \ln \tilde{d}(t)=0
$$

Thus, the correct value of the LCE is obtained (the harmonic oscillator describes a regular oscillatory behaviour, in which trajectories do not converge nor diverge from each other).

If instead we use $\hat{\omega} \neq \omega$, the norm of the perturbation is

$$
\tilde{d}(t)=\frac{\tilde{A}}{L} \sqrt{\sin ^{2}(\omega t+\tilde{\theta})+\frac{\omega^{2}}{\hat{\omega}^{2}} \cos ^{2}(\omega t+\tilde{\theta})}
$$

with $\tilde{A}$ and $\tilde{\theta}$ constants. Although in this case $\tilde{d}(t)$ has an oscillatory behaviour in time, the limit of $1 / t \ln \tilde{d}(t)$ for $t \rightarrow \infty$ is zero, and the correct value of the LCE is once again obtained.

Consider now the finite element discretized equations of a solid continuum. At each time step, the linearized system of Equations (20) can be decoupled into $N$ equations of form (32). Hence, if the characteristic frequency $\hat{\omega}$, used to non-dimensionalize the velocity components, is relatively small (for instance we choose the first natural frequency of the system) then $\omega / \hat{\omega} \gg 1$ for the high-frequency modes and the weight of high-frequency velocity terms in the perturbation norm becomes large.

In a finite element discretization the response associated with high frequency modes is not in general captured by the time discretization (especially when unconditionally stable time integration schemes are used) [16] and it is important to identify the consequences in the LCE calculation. Consider one equation of the form of Equation (32) for the perturbation, i.e. the evolution of one mode of the finite element discretization of a linear system (which cannot be chaotic or unstable). Assume that the chosen characteristic frequency is $\hat{\omega}=1$, the characteristic length is $L=1$, the trapezoidal rule is used, and the time step employed is $\Delta t=0.3 \simeq 2 \pi / 20$ (small enough to capture an oscillation with frequency $\omega=1)$.

The three cases considered below were calculated, and the results are shown in Figure 2.

- $\omega=1$; in this case $\tilde{d}=1$ is obtained, as expected, for every time step, as shown in Figure 2(a).

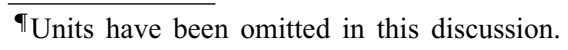



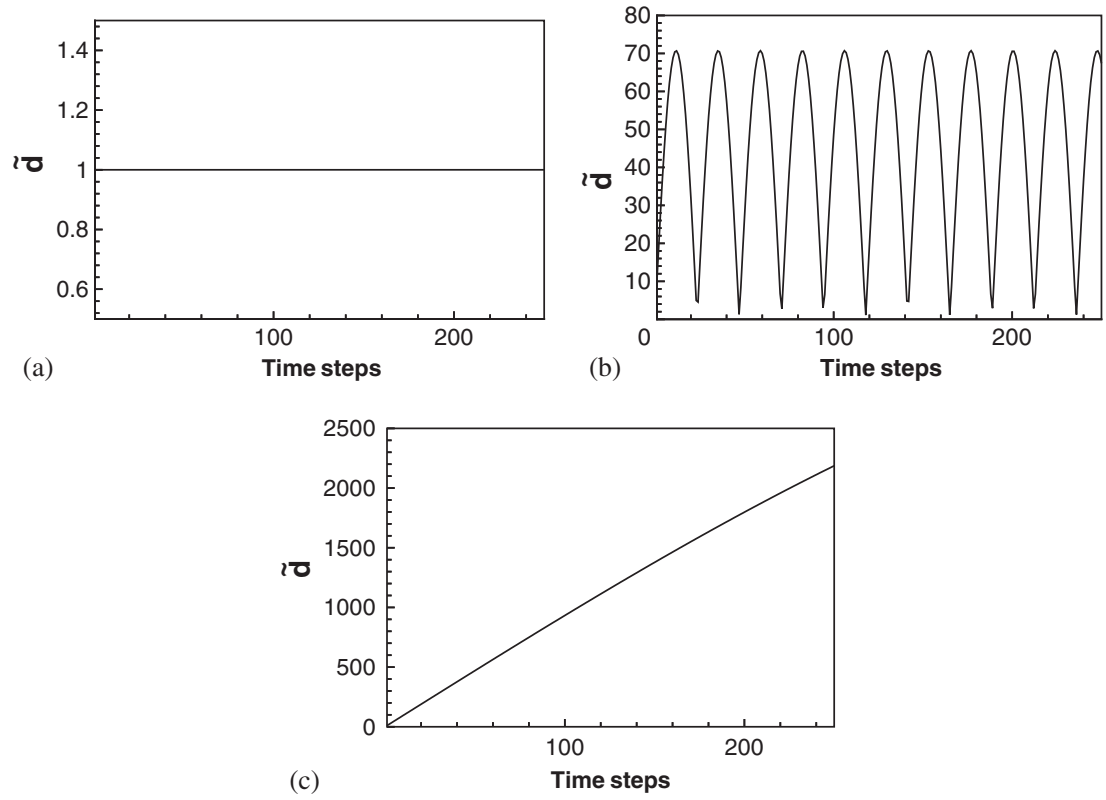

Figure 2. Numerical calculation of the norm of the perturbation, using Equation (29), for the harmonic oscillator equation. The time step used in all three cases is $\Delta t=0.3$ :

(a) $\omega=1$; (b) $\omega=100$; and (c) $\omega=5000$.

- $\omega=100$; Figure 2(b) shows that the calculated value of $\tilde{d}$ is oscillatory, but the amplitude of the oscillations remains constant as a function of time (or time step).

- $\omega=5000$; in this case, shown in Figure 2(c), the norm of the perturbation calculated using Equation (29) grows as a function of the time step. Eventually, the behaviour would be oscillatory but this would only occur after a large number of time steps.

The observed behaviour is due to the time discretization employed, which cannot capture the fast oscillations represented in the equations (when $\omega \gg 1$ ), and is not due to the physics of the problem.

In the case of a finite element discretization, considering finite times, the cumulative effect of the high-frequency modes may induce a growth of the value of $k_{n}$ as a function of time, even when the problem considered is dynamically stable, resulting in the wrong conclusion that the system is unstable.

A solution to this problem is to use a characteristic frequency larger or equal to the maximum frequency, $\omega_{\max }$, of the discretized finite element model, i.e. $\hat{\omega} \geqslant \omega_{\max }$. However, since $\omega_{\max }$ is usually a very large number, in Equation (29) the velocity contributions become negligible, and

$$
\tilde{d}(t) \simeq \frac{\|\tilde{\mathbf{u}}\|}{L}
$$

Thus Equation (36) is used instead of Equation (29). 


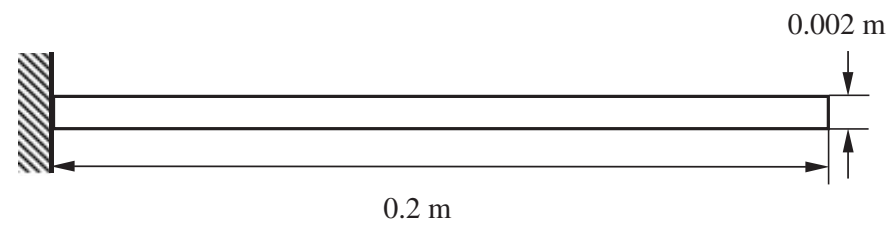

Figure 3. Cantilever beam used in the analysis of the perturbation evolution of a steady-state behaviour.

The use of Equation (36) to assess the exponential divergence of trajectories in the phase space is in fact appropriate for the finite element discretized equations considered here because the velocities are related to the displacements by a time derivative, and any growth of displacements is passed to the velocities (and vice-versa).

Of course, this observation for a structural problem does not hold true in the case of a general system of first-order ordinary differential equations, in which the variables are no longer related to each other by a time derivative.

The behaviour is then unstable if the amplitude of oscillation of $\tilde{d}(t)$ grows in time, and the response LCE is computed as

$$
\chi=\limsup _{t \rightarrow \infty} \frac{1}{t} \ln \tilde{d}(t)
$$

which corresponds to the use of Equations (23)-(26).

\section{EXAMPLES}

In this section the analysis of structural and fluid flow-structure interaction continuous systems is considered. In all cases, the successive approximations to the LCE, $k_{n}$, are calculated using the proposed procedure.

\subsection{Analysis of steady-state behaviour}

This example is included as a validation of the procedure proposed for the calculation of the Lyapunov characteristic exponent of the response of structural continuous systems. The twodimensional plane stress cantilever beam problem shown in Figure 3 is considered. The beam is discretized with 9-node displacement-based elements using a mesh of 100 by 1 elements.

The beam is initially at rest. Since no loading or imposed non-zero displacement boundary conditions are considered, the beam remains at rest. Applying an arbitrary initial perturbation to the system, we expect the perturbation to decay as a function of time if dissipation is included in the model or remain constant if no dissipation is included. Of course, many time integration methods introduce some artificial dissipation in the system response. In this solution, however, the trapezoidal rule, which does not introduce artificial dissipation in the system response, is employed in the calculations [16].

The case in which no dissipation is included in the model and a random perturbation in velocity is applied to the system is considered. Using these initial velocity perturbations, perturbed displacements are calculated and non-dimensionalized. The obtained displacements and velocities are then used as the perturbation initial conditions (such that $d_{0}=1$ ). 


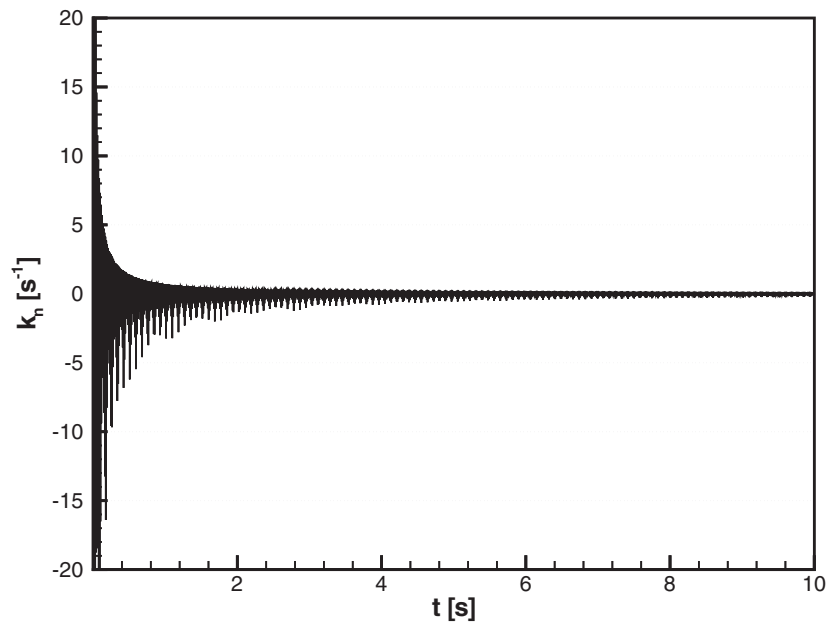

Figure 4. LCE calculation of a steady-state solution of the cantilever beam.

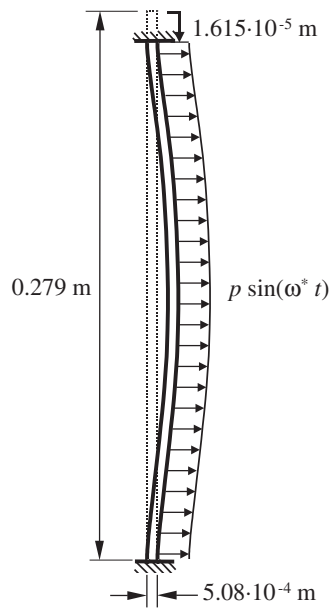

Figure 5. Buckled beam problem considered.

Figure 4 shows a typical curve of successive approximations of the value of the response LCE, $k_{n}$. For this example, a value of zero is expected, and a zero slope is expected in the curve of $\ln \tilde{d}\left(t_{n}\right)=k_{n} t_{n}$ as a function of time. The numerical results correspond to these values (with increasing accuracy as the time step employed, $\Delta t$, is decreased).

\subsection{Buckled beam analysis}

The system shown in Figure 5 is considered. It consists of an initially buckled beam in equilibrium, which is excited with a sinusoidal pressure applied to one of its sides for time 

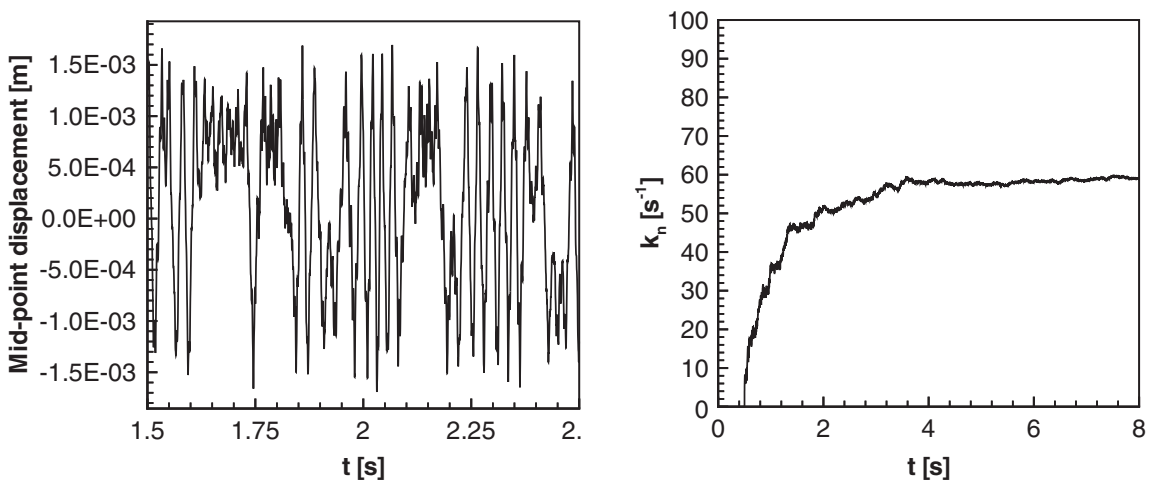

Figure 6. Mid-point displacement of buckled beam problem and LCE calculation.

$t \geqslant 0$. A two-dimensional plane stress analysis, in which large displacements but small strains are assumed, was used in the calculation of the system behaviour. The continuous body was discretized using displacement-based 9-node elements. The system properties and forcing parameters used in the analysis are as follows:

- Young's modulus, $E=206.85 \times 10^{9} \mathrm{~Pa}$

- Poisson's ratio, $v=0.3$

- Density, $\rho=7805.86 \mathrm{~kg} / \mathrm{m}^{3}$

- Excitation amplitude, $p=45 \mathrm{~Pa}$

- Excitation frequency, $\omega^{*}=90 \mathrm{~Hz}$

This type of problem is extensively analysed experimentally in the literature, see for example References [8,19]. The system behaviour can become chaotic under certain values of the forcing amplitude and frequency.

The beam mid-point response and the calculated values of $k_{n}$, which in the limit of $t \rightarrow \infty$ give the response LCE using Equation (26), are shown in Figure 6. The beam mid-point displacement shows a stochastic behaviour with no apparent patterns for positive and negative values (i.e. the beam buckling to one side or the other). The chaotic nature of the system is confirmed by a positive value of the LCE.

A chaotic behaviour is non-periodic and therefore characterized by a response that contains a broad spectrum of frequencies (that is to say, that there are no dominant frequencies but rather all frequencies, in a certain range, are present in the behaviour). The response frequency contents can be extracted by performing a Fourier analysis, see Figure 7 . Note that the spectrum presents a relatively small spike at $90 \mathrm{~Hz}$ (the excitation frequency). Nevertheless a continuous spectrum of frequencies (in which each frequency has approximately the same importance) is otherwise obtained.

Figure 6 shows that in order to obtain a good approximation of the beam response LCE, the behaviour of the perturbations over many time steps need to be calculated. However, the values of $\ln \tilde{d}\left(t_{n}\right)$ provide information about the local stability behaviour (in time) of the system. The values of $\ln \tilde{d}$ as a function of time for the beam response considered are shown in Figure 8. The slope of the curve is positive, indicating an unstable behaviour (associated with growth of perturbations) for the period of time considered. 


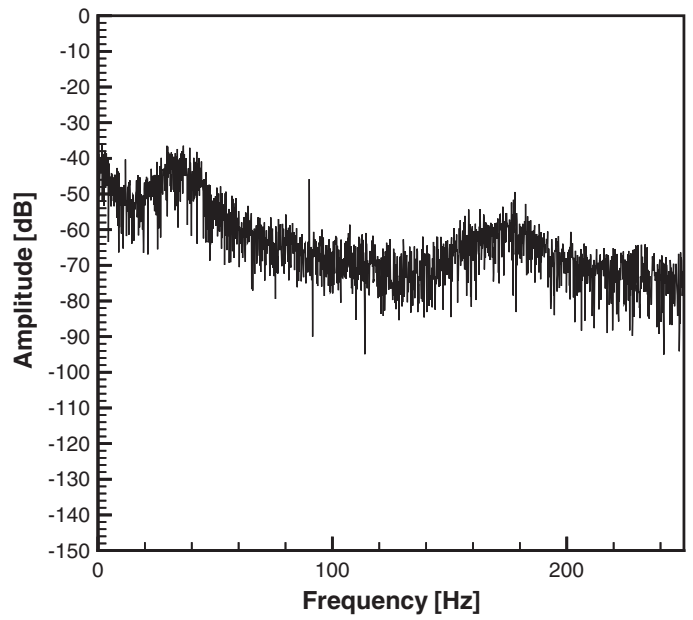

Figure 7. Fourier spectrum of the buckled beam response obtained for the mid-point displacement.

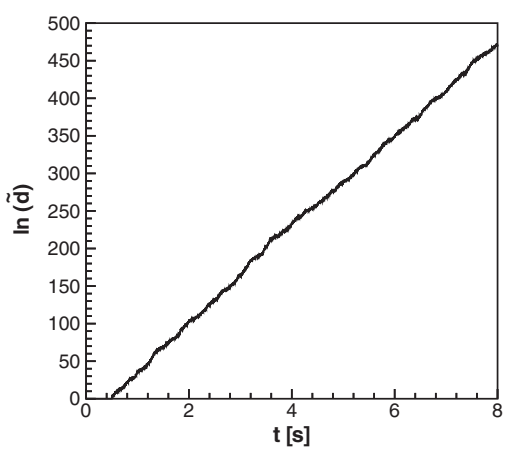

Figure 8. Plot of $\ln \tilde{d}\left(t_{n}\right)$ as a function of time for the buckled beam problem. Note that the local stability of the system is captured by this graph, without explicitly calculating the system LCE (which characterizes the system asymptotic stability).

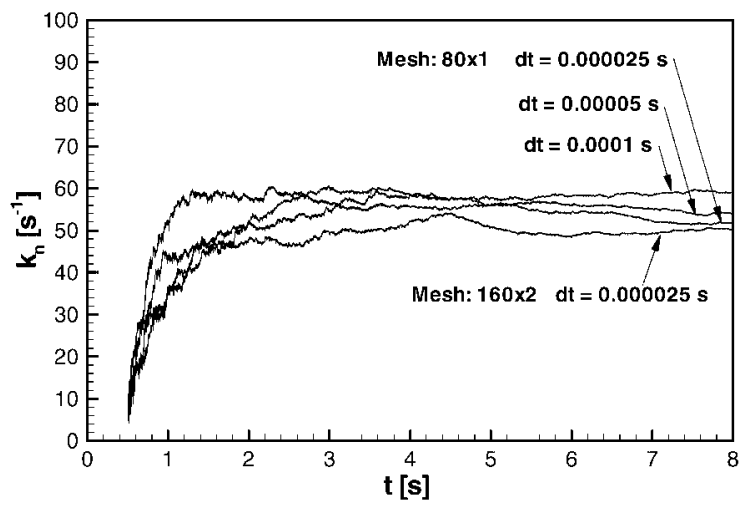

Figure 9. The LCE values obtained in the case of the buckled beam for different solutions.

Convergence of the response LCE is expected as the time step considered is decreased and the mesh used in the discretization is refined. The results of a convergence study for the system are shown in Figure 9. In all cases a chaotic behaviour, characterized by a positive value of the LCE, is detected and the values of $k_{n}$ obtained are all relatively close to each other. 


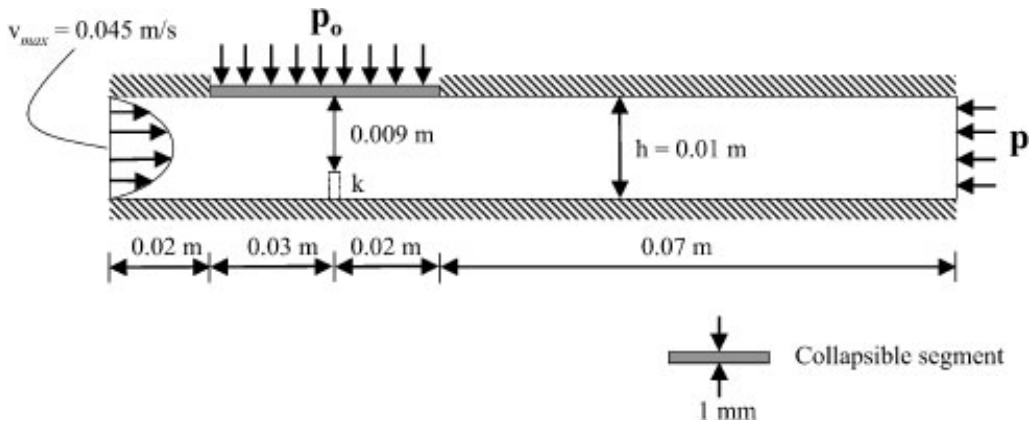

Figure 10. Collapsible channel problem considered. A parabolic velocity profile, constant in time, is imposed at the tube inlet. The linear spring of constant $k$ acts at one point of the membrane when the membrane reaches a vertical displacement of $0.009 \mathrm{~m}$, and it does not interfere with the fluid flow inside the channel.

\subsection{Collapsible channel model}

The fluid flow-structure interaction problem considered is shown in Figure 10. It consists of a two-dimensional channel filled with a viscous fluid. Part of the channel upper wall is a thin structure that can displace in the vertical direction (the collapsible segment). A spring of constant $k$ was included to model a contact condition. The spring starts to act only when the lower point of the collapsible segment, which is originally at $0.05 \mathrm{~m}$ from the channel entrance, has displaced vertically downward by $0.009 \mathrm{~m}$ ( $90 \%$ of the channel height). Thus, a contact non-linear boundary condition is imposed at one point of the collapsible segment. The fluid is modelled using the arbitrary Lagrangian-Eulerian formulation of the almost incompressible Navier-Stokes equations. The fluid domain was discretized using a mesh of 70 by 5 mixed $9 / 3$ velocity/pressure $\left(Q_{2}-P_{1}\right)$ elements [16], whereas the structure was discretized employing 25 by 2 displacement-based 9 -node elements.

This kind of model is used to qualitatively describe the flow of blood inside blood vessels, see for example References [20-22]. A similar fluid-structure interaction model, in which the system behaviour is not chaotic, was also studied by the authors in References [17, 23, 24].

Luo et al. [25] investigated numerically the problem of the collapsible channel. Although in their work a chaotic behaviour was not explicitly found, it was argued that it is possible for the system to develop a chaotic behaviour.

The properties of the system considered are as follows:

- Fluid:

Viscosity, $\mu=0.002 \mathrm{~kg} / \mathrm{m} \mathrm{s}$,

Density, $\rho=1000 \mathrm{~kg} / \mathrm{m}^{3}$,

Bulk modulus, $\kappa=2.1 \times 10^{9} \mathrm{~Pa}$

Pressure difference, $p_{0}-p=15.942 \mathrm{~Pa}$

- Collapsible segment:

$E=2 \times 10^{5} \mathrm{~Pa}$

$v=0.2$

$\rho=1000 \mathrm{~kg} / \mathrm{m}^{3}$

- Linear spring: $k=1000 \mathrm{~kg} / \mathrm{s}^{2}$ 

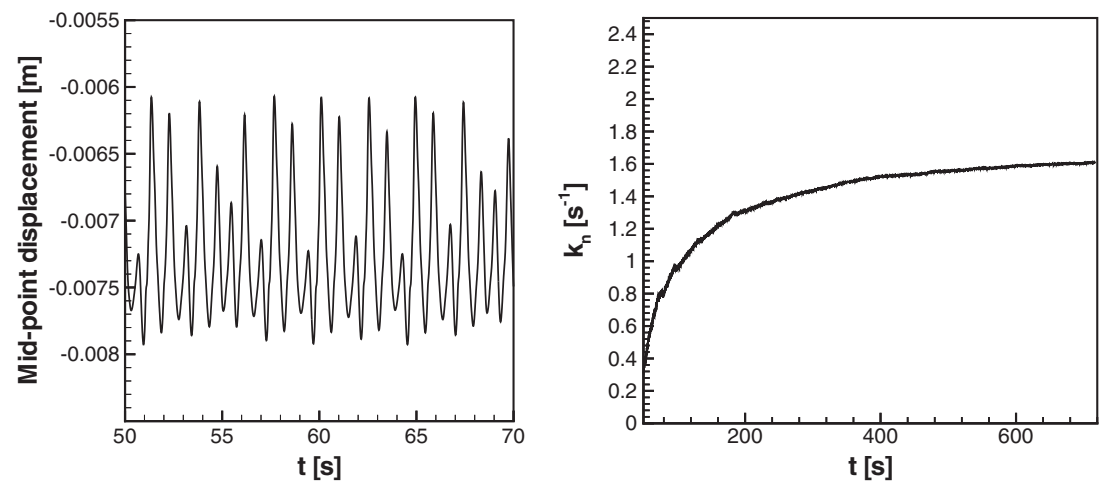

Figure 11. Collapsible segment mid-point displacement for the channel problem together with the calculated values of $k_{n}$.

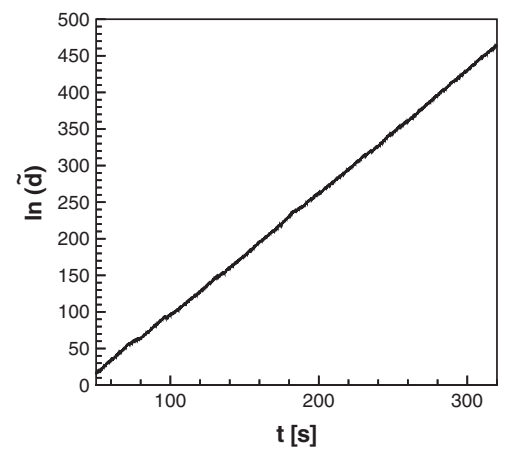

Figure 12. Plot of $\ln (\tilde{d})$ as a function of time for the channel problem.

The obtained long-term system response is shown in Figure 11, together with the calculated values of $k_{n}$. The mid-point displacement response was also computed using ADINA and the same behaviour was obtained (a similar non-periodic motion). It is observed that the segment mid-point response is not periodic, as expected in a chaotic behaviour, and the obtained value of the LCE is positive. Furthermore, the plot of $\ln \tilde{d}$ as a function of time, Figure 12, shows a positive slope for all times considered.

It is interesting to compare the chaotic response solutions in Figures 6 and 11. In the case of the beam the behaviour is highly non-periodic indicating that the divergence of nearby trajectories in phase space is rather strong. In the case considered in Figure 11, even though the displacement is not periodic, it is more 'regular'. Nearby trajectories in phase space diverge from each other exponentially but at a much slower rate than in the case of the beam problem. Hence the LCE obtained for the collapsible channel example is smaller than for the beam problem. The channel problem solution shows a chaotic behaviour that, unlike for the beam problem, is not easily identified by only looking at the system response. Although the successive values of $k_{n}$ converge slowly to a constant value (Figure 11), the local stability indicator already indicates the unstable nature of the response (Figure 12). 


\section{CONCLUDING REMARKS}

In this paper a procedure to calculate the response Lyapunov characteristic exponent (LCE) of structural continuous systems, discretized using finite element methods, is proposed. The procedure is based on the numerical perturbation of all the discrete system degrees of freedom and the calculation of the evolution of the perturbation using the linearized equations of motion at each time step. The procedure can also be applied to fluid flow-structure interaction problems in which the structural part of the system is the focus of the analysis.

Since the response LCE of a system measures the asymptotic divergence of nearby trajectories in phase space, the LCE is a measure of the asymptotic stability of the system response.

A local (temporal) stability indicator is suggested to capture unstable dynamic behaviours shortly after they occur. This indicator should be used after transient effects of the perturbation calculation have decayed.

The analysis of some demonstrative problems has been performed to demonstrate the capabilities of the proposed procedure. The results obtained for the LCE are consistent with the behaviour patterns observed in the system response.

The proposed procedure for the LCE calculation requires only about 25 percent of extra computational time and therefore offers a rather inexpensive way to identify the nature of the dynamic response of non-linear systems.

\section{ACKNOWLEDGEMENT}

The authors are very thankful for the Rocca Fellowship partially supporting this work.

\section{REFERENCES}

1. Guckenheimer J, Holmes P. Nonlinear Oscillations, Dynamical Systems, and Bifurcations of Vector Fields. In Applied Mathematical Sciences, vol. 42. Springer: Berlin, 1983.

2. Argyris J, Faust G, Haase H. An Exploration of Chaos. North-Holland: Amsterdam, 1994.

3. Strogatz SH. Nonlinear Dynamics and Chaos. Addison-Wesley: New York, 1994.

4. Benettin G, Galgani L, Strelcyn JM. Kolmogorov entropy and numerical experiments. Physical Review A 1976; 14:2338-2345.

5. Benettin G, Galgani L, Giorgilli A, Strelcyn JM. Lyapunov characteristic exponents for smooth dynamical systems and for Hamiltonian systems: a method for computing all of them. Part 1: Theory. Part 2: Numerical application. Meccanica 1980; 15:9-30.

6. Geist K, Parlitz U, Lauterborn W. Comparison of different methods for computing Lyapunov exponents. Progress of Theoretical Physics 1990; 83:875-893.

7. Moon FC. Chaotic Vibrations. Wiley: New York, 1987.

8. Lacarbonara W, Nayfeh AH. Experimental validation of reduction methods for non-linear vibrations of distributed-parameter systems: analysis of a buckled beam. Nonlinear Dynamics 1998; 17:95-117.

9. Moon FC. Experimental measurement of chaotic attractors in solid media. Chaos 1991; 1:31-41.

10. Moon FC, Shaw SW. Chaotic vibrations of a beam with non-linear boundary conditions. International Journal of Non-linear Mechanics 1983; 18:465-477.

11. Païdoussis MP, Li GX, Moon FC. Chaotic oscillations of the autonomous system of a constrained pipe conveying fluid. Journal of Sound and Vibration 1989; 135:1-19.

12. Argyris J, Tenek L. Nonlinear and chaotic oscillations of composite plates and shells under periodic heat load. Computer Methods in Applied Mechanics and Engineering 1995; 122:351-377.

13. Argyris J, Tenek L, Andreadis I, Athanasiou M, Pavlos G. On chaotic oscillations of a laminated composite cylinder subject to periodic application of temperature. Chaos, Solitons and Fractals 1998; 9:1529-1554.

14. Oseledec VI. A multiplicative ergodic theorem. Ljapunov characteristic numbers for dynamical systems. Transactions of the Moscow Mathematical Society 1968; 19:197-231. 
15. Haken H. At least one Lyapunov exponent vanishes if the trajectory of an attractor does not contain a fixed point. Physics Letters A 1983; 94:71-72.

16. Bathe KJ. Finite Element Procedures. Prentice Hall: Englewood Cliffs, NJ, 1996.

17. Rugonyi S, Bathe KJ. On the Analysis of fully-coupled fluid flows with structural interactions - a coupling and condensation procedure. International Journal for Computational Civil and Structural Engineering 2000; 1: $29-41$.

18. Rugonyi S, Bathe KJ. On finite element analysis of fluid flows fully coupled with structural interactions. Computer Modeling in Engineering and Sciences 2001; 2:79-96.

19. Kreider W, Nayfeh AH. Experimental investigation of single-mode response in a fixed-fixed buckled beam. Nonlinear Dynamics 1998; 15:155-177.

20. Pedley TJ. The Fluid Mechanics of Large Blood Vessels. Cambridge University Press: Cambridge, 1980.

21. Lowe TW, Pedley TJ. Computation of Stokes flow in a channel with a collapsible segment. Journal of Fluids and Structures 1995; 9:885-905.

22. Luo XY, Pedley TJ. A numerical simulation of steady flow in a 2-D collapsible channel. Journal of Fluids and Structures 1995; 9:149-174.

23. Rugonyi S, Bathe KJ. Lyapunov characteristic exponent calculation for finite element discretized models. In Computational Fluid and Solid Mechanics, Bathe KJ (ed.), Elsevier Science: Amsterdam, 2001.

24. Bathe KJ, Rugonyi S, De S. On the current state of finite element methods - solids and structures with full coupling to fluid flows. Plenary Lecture Proceedings of the 4th International Congress on Industrial and Applied Mathematics (ICIAM 99). Oxford University Press: Oxford, 1999.

25. Luo XY, Pedley TJ. A numerical simulation of unsteady flow in a two-dimensional collapsible channel. Journal of Fluid Mechanics 1996; 314:191-225. 